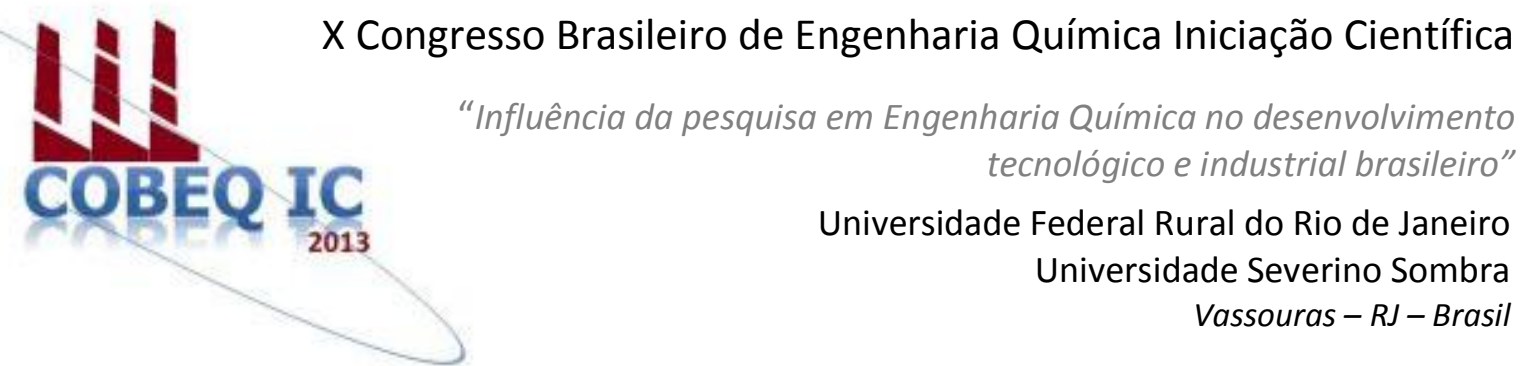

\title{
RECUPERAÇÃO DE PRATA DE RESÍDUOS FOTOGRÁFICOS POR ELETRODEPOSIÇÃO
}

\author{
PRADO**1 , P. F. A.; BRITTO-COSTA ${ }^{2}$, P. H.; RUOTOLO ${ }^{3}$, L. A. M. \\ ${ }^{1}$ Aluno do DEQ/UFSCar ${ }^{2}$ Doutorando do DEQ/UFSCar ${ }^{3}$ Professor do DEQ/UFSCar \\ Departamento de Engenharia Química - Universidade Federal de São Carlos \\ Endereço-UFSCar, SP 310, Rod. Washington Luis km 235, São Carlos, CEP. 13.565-905, SP, \\ email: pluis@ufscar.br
}

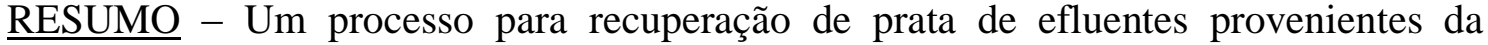
indústria fotográfica foi estudado. Primeiramente realizou-se um estudo voltamétrico de solução contendo íons prata e posteriormente, de uma solução real de revelação fotográfica. Na etapa de eletrorrecuperação em reator eletroquímico, foi observado que para potenciais de $-450 \mathrm{mV} v s \mathrm{Ag} / \mathrm{AgCl}$ houve deposição de prata porém ao alcançar-se potenciais muito negativos, ocorre o desprendimento de hidrogênio decorrente da reação de redução da água e a formação de $\mathrm{Ag}_{2} \mathrm{~S}$, depositando-se na superfície do eletrodo. A qualidade do depósito e eficiência do processo depende das condições de controle da corrente a fim de evitar-se a formação de $\mathrm{Ag}_{2} \mathrm{~S}$. Modulou-se então a corrente mantendo-se valores de corrente limite, a qual foi calculada conhecendo-se o coeficiente de transporte de massa, determinado experimentalmente. Os resultados mostraram que é possível recuperar prata de modo eficiente, evitando-se reações paralelas, quando se utiliza a solução simulada. No entanto, para a solução real houve eletrodeposição efetiva, indicando que as condições operacionais devem ser otimizadas para a aplicação da corrente modulada ao processo.
\end{abstract}

Palavras chave: eletrodeposição, prata, corrente limite, sustentabilidade.

\section{INTRODUÇÃO}

\section{Processo Fotográfico}

O processo de revelação fotográfica, descoberto em 1837, têm sido aplicado em larga escala nos últimos 200 anos. O processo convencional de fixação envolve a utilização de soluções de tiossulfato de sódio (TSS) para dissolução de haletos de prata ( $\mathrm{AgX}$, conhecido como gerador de cor) (El-Sattar e El-Shayeb, 2004). A reação envolvida no processo é descrita pela Equação 1.

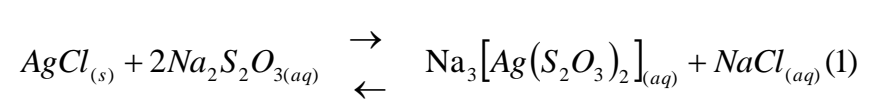

Os banhos de fixação, como são chamadas as emulsões resultantes do processo de revelação fotográfica, contêm um grande número de outros produtos químicos além dos haletos de prata. Produtos como ácidos, conservantes, endurecedores e tampões, criam um meio bastante complexo (Adani et al., 
2005). Uma composição típica desse banho contém concentração de prata de $4050 \mathrm{mg} \mathrm{L}^{-1}$, muito acima do valor permitido para descarte em rede de esgoto $\left(1,5 \mathrm{mg} \mathrm{L}^{-1}\right)$ determinado pela CETESB.

\section{Recuperação de metais}

Devido às restrições ambientais e à crescente rigor no monitoramento dos efluentes industriais contendo íons metálicos, novas tecnologias são necessárias para garantir o descarte correto desses efluentes.

$\mathrm{O}$ tratamento de águas residuárias contendo íons metálicos é comumente feito por precipitação com bases como $\mathrm{NaOH}$ ou $\mathrm{CaO}$ e posterior sedimentação. Esse processo no entanto gera poluição secundária ao utilizar-se compostos químicos e cria a necessidade de disposição de uma lama residuária final. O tratamento por troca iônica, utilizando resinas catiônicas ou zeólitas, também é aplicado, porém transfere o metal da fase aquosa para a fase sólida. $\mathrm{O}$ armazenamento do resíduo e seu transporte tornam-se etapas custosas e tornam o processo pouco eficiente do ponto de vista econômico e ambiental.

A tecnologia eletroquímica aplicada à recuperação de metais permite um processo com controle automatizado e de baixo custo, já que não há gastos com o pós tratamento, transporte ou estocagem de intermediários ou resíduos, como ocorre com os métodos de recuperação de metais mencionados anteriormente. Ademais, o preço da energia elétrica frente aos gastos atrelados à compra e disposição de reagentes químicos que seriam usados para esse fim, encontra-se bastante competitivo atualmente. A prata recuperada eletroquimicamente apresenta-se na sua forma mais pura e possui um alto valor agregado, justificando sua recuperação. $\mathrm{O}$ processo ainda entra na área de tecnologias "limpas" pois evita o uso de compostos químicos, utilizando o elétron como reagente.

\section{Voltametrias Cíclicas}

As varreduras de potencial em soluções resultam em densidades de corrente específicas a cada potencial, decorrentes de transferência de elétrons no meio, na forma de um voltamograma com picos. Os voltamogramas revelam, portanto, a ocorrência de reações de oxidação e redução como também a variedade de espécies eletroativas. A forma, potencial, densidade de corrente, cargas associadas e balanço de cargas entre picos revelam algumas características desses processos de oxi-redução. A diferença entre ciclos de varredura também pode fornecer informações quanto à estabilidade de componentes e reversibilidade das reações.

\section{MATERIAIS E MÉTODOS}

\section{Sistema experimental}

O sistema utilizado para recuperação de prata está representado na Figura 1.

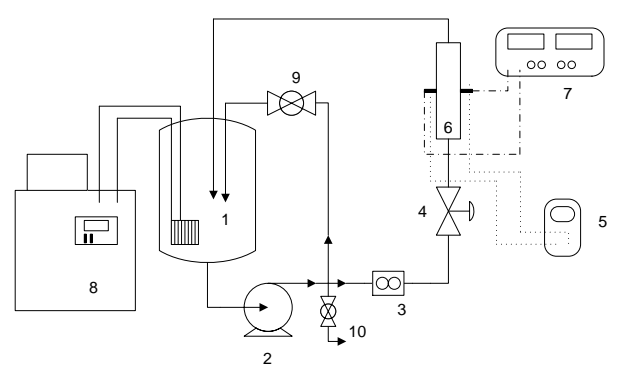

Figura 1. Sistema experimental.

$\mathrm{Na}$ Figura 1, tem-se: 1) tanque de eletrólito 5L; 2) bomba centrífuga; 3) rotâmetro; 4) válvula diafragma para controle e regulagem de vazão; 5) voltímetro para medida do potencial de célula; 6) reator eletroquímico; 7) fonte de corrente/potenciostato; 8) banho termostático; 9) válvula esfera (by-pass); 10) válvula esfera (esgotamento)

O reator eletroquímico (6), é composto por um cátodo poroso de CVR (carbono vítreo reticulado), cuja porosidade é de 60 ppi e a área é de $1166 \mathrm{~m}^{2} \mathrm{~m}^{-3}$. Posteriormente, utilizou-se para o cátodo uma placa plana de aço inox de área $0,07 \mathrm{~m}^{2}$. O ânodo utilizado foi de $\mathrm{Ti} / \mathrm{Ti}_{0,7} \mathrm{Ru}_{0,3} \mathrm{O}_{2}$. Nos experimentos de voltametria cíclica utilizou-se uma placa plana de platina $\left(0,07 \mathrm{~m}^{2}\right)$ como eletrodo de trabalho. O eletrodo de referência utilizado foi o de $\mathrm{Ag} / \mathrm{AgCl}$.

\section{Determinação do coeficiente de difusão da prata}

A determinação da constante de difusão da prata no meio aquoso foi realizada 
utilizando-se uma célula contendo uma membrana porosa (diafragma), em equipamento mostrado na Figura 2.

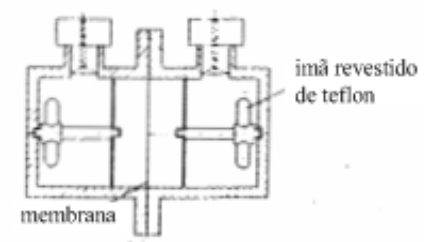

(a)

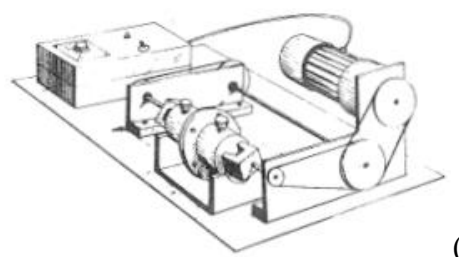

(b)

Figura 2. Célula de difusão: (a) visão interna e (b) vista explodida

Introduzindo-se duas soluções de concentrações distintas de prata e ligando-se o sistema de polias, os dois compartimentos eram agitados por um tempo determinado. Retirava-se as amostras e analisava-se, por espectroscopia de absorção, as concentrações finais e iniciais de cada compartimento. $\mathrm{O}$ coeficiente de difusão foi calculado utilizandose a Equação 2.

$$
\ln \left[\frac{\left(C_{A f}^{1}-C_{A f}^{2}\right)}{\left(C_{A 0}^{1}-C_{A 0}^{2}\right)}\right]=-\beta D_{A B} t
$$

A constante de célula $\beta$ foi determinada através de experimentos com $\mathrm{KCl}$ (Gubulin e Tobinaga, 1990).

\section{RESULTADOS E DISCUSSÕES}

\section{Caracterização do efluente}

$\mathrm{O}$ efluente real foi caracterizado quanto à sua concentração de prata através de espectroscopia de absorção atômica. Obteve-se uma concentração de $\mathrm{Ag}^{+}=800 \mathrm{ppm}$. Mediuse também a condutividade e o $\mathrm{pH}$ do meio, obtendo-se valores de $35,8 \mathrm{mS} \mathrm{cm}^{-1}$ e 7,3, respectivamente. A concentração de $\mathrm{Na}_{2} \mathrm{~S}_{2} \mathrm{O}_{3}$ comumente encontrada em efluentes reais é de 8,75 $\mathrm{g} \mathrm{L}^{-1}$, de acordo com Adani et al., 2005. A solução simulada foi preparada então com base nesses valores.

\section{Análise inicial: Voltametrias cíclicas}

$\mathrm{O}$ efluente real e a solução simulada foram caracterizados eletroquimicamente por voltametrias cíclicas em duas faixas de potencial diferentes. Na Figura 3, observa-se, no caso da solução sintética, que há o aparecimento de um pico catódico em $-0,4 \mathrm{~V}$ referente ao processo de eletrodeposição de prata e outro pico em aproximadamente $0,2 \mathrm{~V}$ referente à oxidação da prata eletrodepositada. Para o efluente real, além dos dois picos referentes à deposição e dissolução da prata, observa-se também um outro pico anódico em $0,1 \mathrm{~V}$, indicando que outras espécies presentes na solução estão sendo oxidadas.

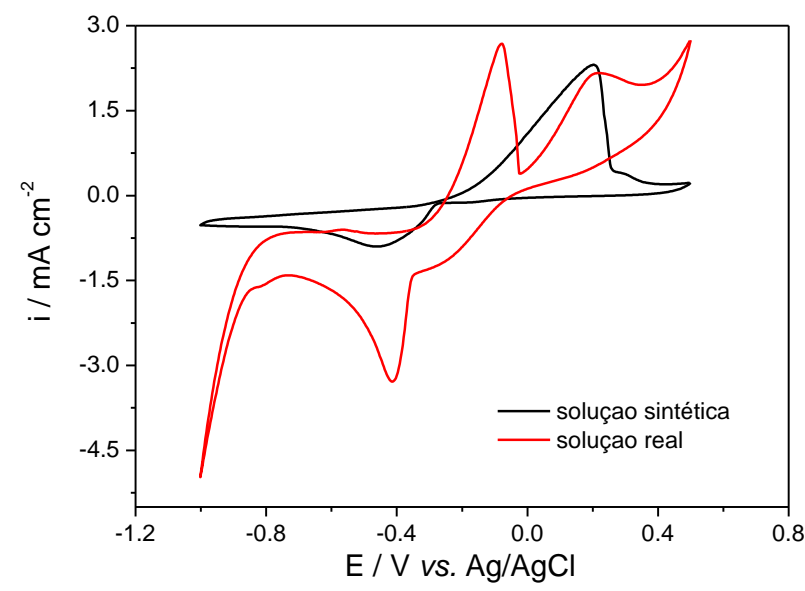

Figura 3. Voltamograma da solução real e sintética. $50 \mathrm{mV} \mathrm{s}^{-1}$. Placa plana Pt $1 \mathrm{~cm} \mathrm{x} 1$ $\mathrm{cm}$.

Estendeu-se a faixa de potencial analisada para valores entre $-1,5 \mathrm{~V}$ e $1,0 \mathrm{~V}$ (Figura 4). Neste caso, ocorre o surgimento de um novo pico de oxidação no potencial de 0,7 $\mathrm{V}$, atribuído à formação de $\mathrm{Ag}_{2} \mathrm{~S}$, que ocorre em potenciais muito catódicos, já que se observou um depósito preto e pulverolento. A presença de $\mathrm{Ag}_{2} \mathrm{~S}$ foi posteriormente confirmada por difração de raios-X. 


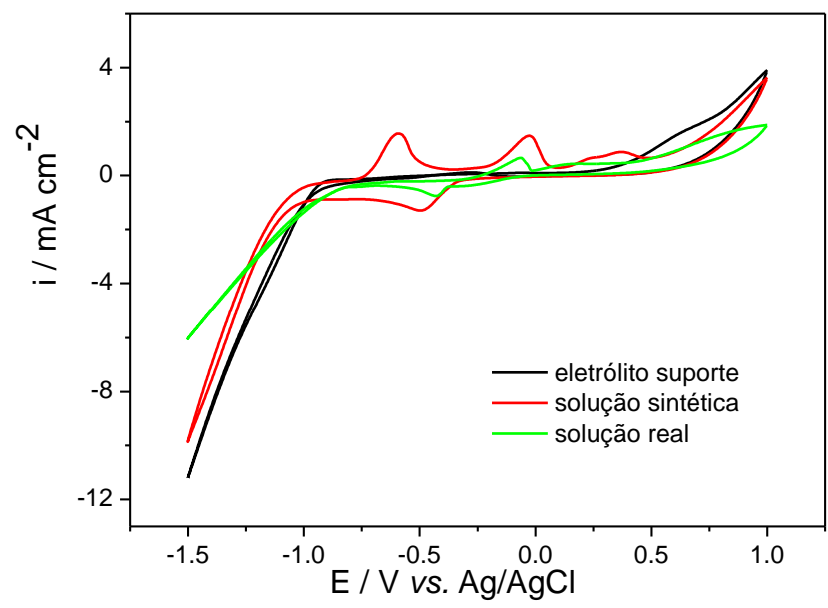

Figura 4. Voltamograma da solução real, sintética e suporte. $50 \mathrm{mV} \mathrm{s}^{-1}$. Pt $1 \mathrm{~cm} \mathrm{x} 1 \mathrm{~cm}$.

\section{Eletrodeposição}

Em um primeiro experimento de eletrodeposição aplicou-se $120 \mathrm{~mA}$ por 70 minutos e praticamente toda a prata foi eletrodepositada sobre a superfície do eletrodo. A Figura 5 mostra a concentração de prata normalizada em função do tempo. O consumo energético e a eficiência de corrente foram de $6,42 \mathrm{kWh} \mathrm{m}^{-3}$ e $155 \%$, respectivamente. O consumo energético é baixo, principalmente comparado ao preço da prata metálica. O valor de eficiência acima de $100 \%$ revela que outros processos de consumo de prata no meio estão ocorrendo

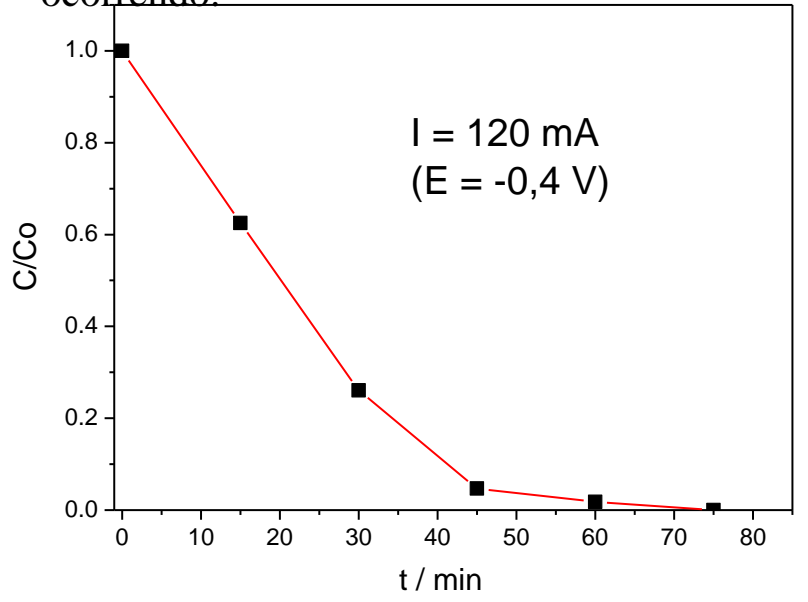

Figura 5. Teste cinético em reator eletroquímico. Eletrodo CVR, vazão: $3 \mathrm{~L} \mathrm{~min}^{-1}$

\section{Efeito do pH}

Durante o processo de eletrólise, o $\mathrm{pH}$ do meio sofre alteração devido ao aumento da concentração de íons $\mathrm{H}^{+}$devido à eletrólise da água. A Figura 6 mostra voltametrias cíclicas da solução de prata medidas em soluções com diferentes valores $\mathrm{pH}$.

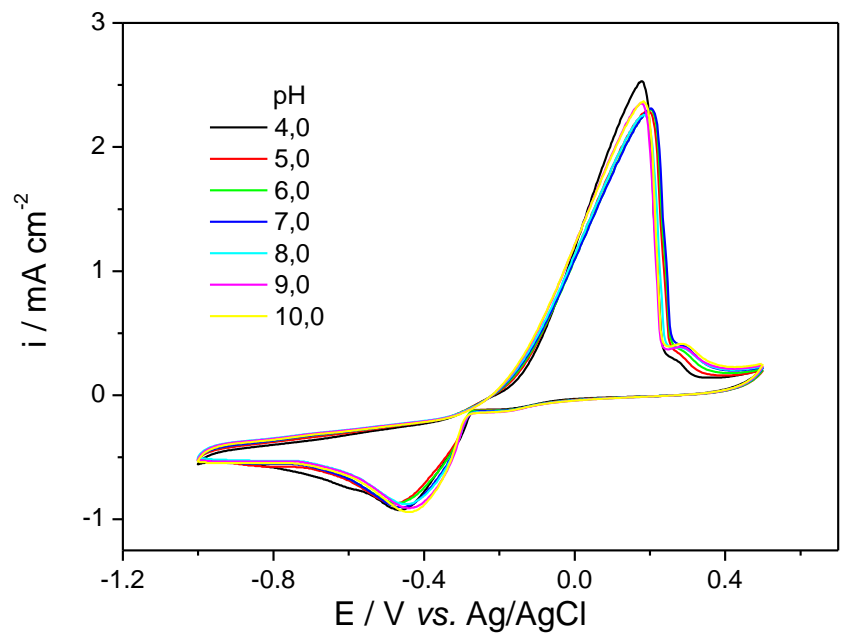

Figura 6. Voltamogramas da solução de prata em diferentes pHs. $50 \mathrm{mV} \mathrm{s}^{-1}$. Pt $\left(1 \mathrm{~cm}^{2}\right)$.

$\left[\mathrm{Ag}^{+}\right]=50 \mathrm{ppm} ;\left[\mathrm{Na}_{2} \mathrm{~S}_{2} \mathrm{O}_{3}\right]=8,75 \mathrm{~g} \mathrm{~L}^{-1}$.

Observa-se que os potenciais de oxiredução não se alteram com a mudança de $\mathrm{pH}$. Para $\mathrm{pH}<4$ houve precipitação de um sólido branco, identificado como enxofre proveniente dos íons $\mathrm{S}_{2} \mathrm{O}_{3}{ }^{2-}$.

\section{Processo de filtração}

Observada a formação de enxofre a baixos pHs e sabendo-se que a geração de $\mathrm{Ag}_{2} \mathrm{~S}$ está diretamente relacionada à presença de enxofre no meio, foram realizados testes em paralelo para remoção do enxofre no meio reduzindo-se o $\mathrm{pH}$ e filtrando-se a suspensão resultante com papel de filtro qualitativo. Foram feitas voltametrias cíclicas desta solução, no entanto houve o aparecimento do precipitado preto de $\mathrm{Ag}_{2} \mathrm{~S}$. Utilizou-se então um filtro quantitativo (membrana de acetato de celulose com porosidade de $0,45 \mu \mathrm{m})$. Realizou-se novamente uma voltametria cíclica da solução microfiltrada, como mostrado na Figura 7. 


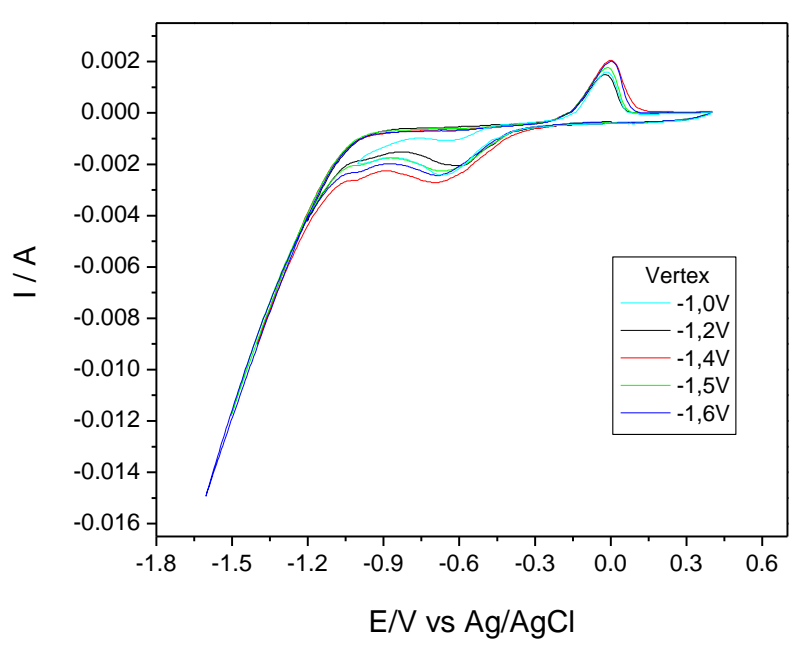

Figura 7. Voltamogramas da solução microfiltrada em função do potencial do vértex catódico. $\left[\mathrm{Ag}^{+}\right]=400 \mathrm{ppm} ; \sigma=9,82 \mathrm{mS} \mathrm{cm}$.

$$
50 \mathrm{mV} \mathrm{s}^{-1} \text {. }
$$

Não se observa na Figura 7 nenhum pico de corrente em torno de $-0,7 \mathrm{~V}$, o que corresponderia à formação de $\mathrm{Ag}_{2} \mathrm{~S}$ ).

Uma nova eletrólise foi realizada aplicando-se $3 \mathrm{~mA}$ e uma concentração inicial de prata menor (200 ppm), a qual corresponde à faixa de concentração de prata mais crítica em relação à formação de $\mathrm{Ag}_{2} \mathrm{~S}$. As características da solução microfiltrada e da solução pós-eletrólise são mostradas na Tabela 1 .

Tabela 1. Características da solução antes e após as eletrólises aplicando-se $3 \mathrm{~mA}$; $\mathrm{Pt}\left(1 \mathrm{~cm}^{2}\right)$.

\begin{tabular}{ccc}
\hline & $\begin{array}{c}\text { Solução } \\
\text { Microfiltrada }\end{array}$ & $\begin{array}{c}\text { Solução Final após } \\
\text { eletrodeposição }\end{array}$ \\
\hline$[\mathrm{Ag}+] / \mathrm{ppm}$ & 374 & 18,5 \\
$\mathrm{pH}$ & 3,5 & 4,6 \\
$\sigma / \mathrm{mS} \mathrm{cm}^{-1}$ & 9,82 & 10,08 \\
\hline
\end{tabular}

A diminuição da concentração observada na Tabela 1, de 374 ppm para 18,5 ppm em 2 horas de experimento revela que houve significativa remoção de prata da solução. Um depósito cinza escuro foi observado e para averiguar sua natureza, realizou-se uma análise de difratometria de raios-X (DRX) do depósito, mostrado na Figura 8.

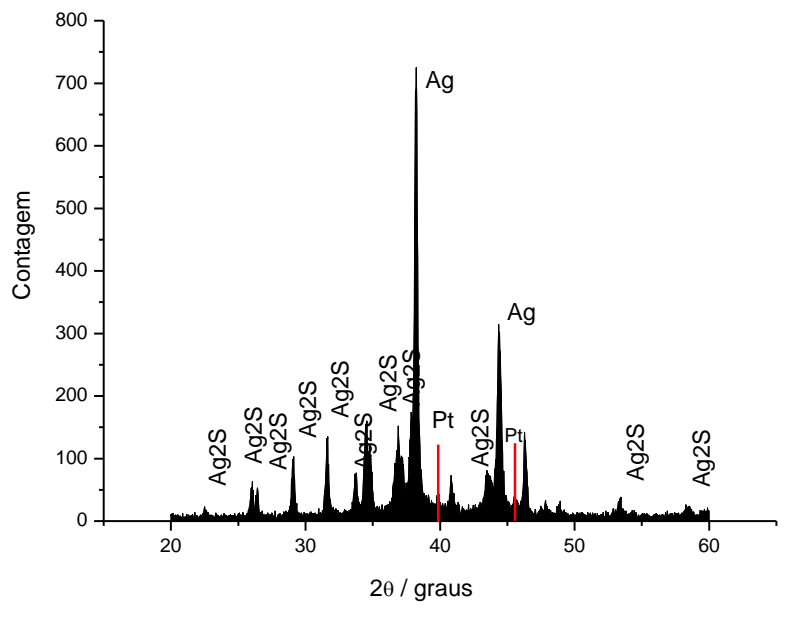

Figura 8. DRX do depósito de prata obtido na eletrólise a $3 \mathrm{~mA}$. Eletrólito microfiltrado.

Além do substrato de platina utilizado, há dois picos intensos, referentes à prata eletrodepositada, porém há também picos indicando a formação de $\mathrm{Ag}_{2} \mathrm{~S}$; portanto conclui-se que os processos de precipitação e microfiltração não foram eficientes para remover todo o $\mathrm{S}_{2} \mathrm{O}_{3}{ }^{2-}$ da solução.

\section{Coeficiente de transporte de massa}

A estratégia de modulação da corrente consiste em conhecer a corrente limite em função do tempo de operação, supondo-se eficiência de corrente máxima para o processo (nesse caso, supôs-se 100\%). A densidade de corrente limite, dada pela Equação 3, é expressa em função do tempo, $\mathrm{C}=\mathrm{C}(\mathrm{t})$, sendo $\mathrm{C}$ a concentração de prata.

$$
i_{\text {lim }}=\frac{z \cdot F}{M} \cdot k_{m} \cdot C
$$

O coeficiente de transporte de massa $\left(\mathrm{k}_{\mathrm{m}}\right)$ é expresso na Equação 4 como uma relação entre o coeficiente de difusão da prata $\left(\mathrm{D}_{\mathrm{Ag}+}\right)$ pela espessura da camada limite $(\delta)$ :

$$
k_{m}=\frac{D_{A g+}}{\delta}
$$

O coeficiente de difusão dos íons prata, $6,410^{-11} \mathrm{~m}^{2} \mathrm{~s}^{-1}$, foi determinado através do experimento na célula de difusão e a espessura da camada limite, $1,6110^{-10} \mathrm{~m}$ foi obtido de correlações existentes na literatura (Beraldo, 2011). O coeficiente de transporte de massa 
foi então calculado, sendo seu valor de 3,97 $10^{-1} \mathrm{~m} \mathrm{~s}^{-1}$.

\section{Eletrólise com a corrente modulada}

Solução simulada: Para a solução simulada, observa-se na Figura 9 que a prata foi totalmente removida por eletrodeposição após 210 minutos e que os valores das concentrações de prata calculadas e medidas foram próximos. O comportamento observado é típico de um processo controlado por transporte de massa, confirmando que o processo está ocorrendo em cinética de corrente limite.

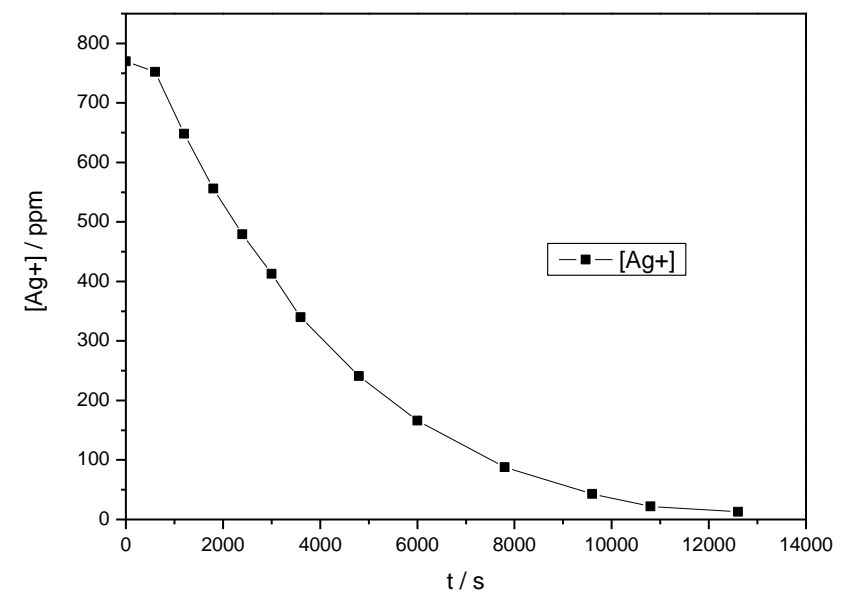

Figura 9. Concentração de prata em função do tempo. $\left[\mathrm{Ag}^{+}\right]_{0}=770 \mathrm{ppm} ;\left[\mathrm{Na}_{2} \mathrm{~S}_{2} \mathrm{O}_{3}\right]=8,75 \mathrm{~g}$ $\mathrm{L}^{-1} ; \mathrm{V}=0,5 \mathrm{~L}$; aço inox $0,07 \mathrm{~m}^{2}$.

A eficiência de corrente observada foi próxima de $100 \%$, como mostra a Tabela 2 . Na Figura 10 constata-se visualmente que não houve a formação de $\mathrm{Ag}_{2} \mathrm{~S}$.

Tabela 2. Eficiência de corrente do processo de eletrodeposição usando efluente simulado

\begin{tabular}{cccccc}
\hline $\mathbf{t} / \mathbf{m i n}$ & $\mathbf{I}_{\mathbf{L}} / \mathbf{A}$ & $\begin{array}{c}\left.\mathbf{A g}^{+}\right] / \mathbf{m g ~ L}^{-1} \\
\text { (calculada) }\end{array}$ & $\mathbf{E} / \mathbf{V}$ & $\begin{array}{c}\left.\mathbf{A g}^{+}\right] / \mathbf{m g ~ L}^{-1} \\
\text { (medida) }\end{array}$ & $\mathbf{E C ~ / \%}$ \\
\hline 0 & 0,085 & 770 & $-2,24$ & 770 & - \\
30 & 0,054 & 494 & $-1,88$ & 556 & 109 \\
60 & 0,035 & 317 & $-1,78$ & 340 & 134 \\
100 & 0,019 & 176 & $-1,66$ & 166 & 107 \\
130 & 0,012 & 113 & $-1,65$ & 88 & 100 \\
160 & 0,008 & 72 & $-1,62$ & 43 & 90
\end{tabular}

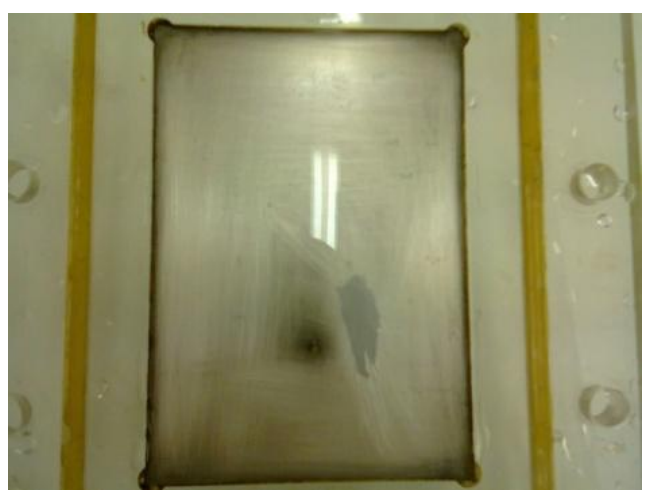

Figura 10. Superfície do eletrodo de aço inox após o experimento de eletrodeposição.

Solução real: ao realizar-se o experimento de eletrodeposição com o efluente real observouse que a solução, bastante escura, não teve sua concentração de prata modificada ao longo do tempo de eletrólise, como mostra a Tabela 3.

Tabela 3. Eficiência de corrente do processo de eletrodeposição usando efluente

\begin{tabular}{ccccc}
\multicolumn{5}{c}{ real } \\
\hline $\mathbf{t} / \mathbf{m i n}$ & $\mathbf{I L} / \mathbf{A}$ & $\begin{array}{c}{\left[\mathbf{A g}^{+}\right] / \mathbf{m g ~ L} \mathbf{~}^{-1}} \\
\text { (calculada) }\end{array}$ & $\mathbf{E} / \mathbf{V}$ & $\begin{array}{c}{\left[\mathbf{A g}^{+}\right] / \mathbf{m g ~ L}^{-1}} \\
\text { (medida) }\end{array}$ \\
\hline 0 & 0,107 & 970 & $-0,85$ & 886 \\
30 & 0,069 & 623 & $-0,64$ & 462 \\
60 & 0,044 & 400 & $-0,49$ & 465 \\
100 & 0,024 & 221 & $-0,38$ & 457 \\
130 & 0,016 & 142 & $-0,31$ & 553 \\
160 & 0,010 & 91 & $-0,24$ & 560
\end{tabular}

Acredita-se que esse resultado seja devido à aplicação de baixos valores de corrente decorrentes de um valor subestimado de $\mathrm{D}_{\mathrm{Ag}+}$, o qual foi determinado utilizando-se o eletrólito simulado.

\section{CONCLUSÃO}

O valor do coeficiente de difusão determinado pela técnica da célula de difusão foi bastante satisfatório para a determinação de $\mathrm{k}_{\mathrm{m}}$ e da corrente limite no caso da solução simulada, permitindo a obtenção de um depósito isento de $\mathrm{Ag}_{2} \mathrm{~S}$ e altos valores de eficiência de corrente (próximos a 100\%) para a eletrodeposição de prata em reator eletroquímico. No entanto, para a solução real, não houve eletrodeposição satisfatória devido à complexidade dos compostos do meio. 


\section{NOTAÇÃO}

I corrente, A

$\mathrm{I}_{\mathrm{L}} \quad$ corrente limite, A

i densidade de corrente limite, $\mathrm{mA} \mathrm{cm}^{-2}$

$\mathrm{E}$ potencial, $\mathrm{V}$

$\sigma \quad$ condutividade, $\mathrm{mS} \mathrm{cm}^{-1}$

$\mathrm{D}_{\mathrm{Ag}+} \quad$ constante de difusão, $\mathrm{m}^{2} \mathrm{~s}^{-1}$

$\delta \quad$ espessura da camada limite, $\mathrm{m}$

$\mathrm{k}_{\mathrm{m}} \quad$ coeficiente de difusão $\mathrm{m} \mathrm{s}^{-1}$

\section{BIBLIOGRAFIA}

ADANI, K. G. BARLEY, R. W.; PASCOE, R. D. (2005). Silver recovery from synthetic photographic and medical X-ray process effluents using activated carbon. Min. Eng. 18, 1269-1276.

EL-SATTAR, A. A.; EL-SHAYEB, M.; DIEF, H. A. (2004). Silver recovery from photographic and X-ray films and fixer solution waste using a packed bed reactor. Alex. Eng. J., 43(3), 393-399.

GUBUlin, J. C.; TOBINAGA, S. (1990). Transferência de Massa. In Gubulin, J.C.; Freire J.T. "Tópicos Especiais em Fenômenos de Transporte". EDUFSCAR, São Carlos, 1990. p. 134-144.

BERALDO, R. (2011). Degradação de compostos fenólicos presentes em efluentes da indústria do petróleo. Universidade Federal de São Carlos, São Carlos, São Carlos.

\section{AGRADECIMENTOS}

Agradecimentos à FAPESP, CNPq e DEQ/UFSCar pelo apoio durante o projeto e a bolsa de iniciação tecnológica ao aluno. 